\title{
Faddeev-type calculation of three-body nuclear reactions including core excitation
}

\author{
A. Deltuva \\ Centro de Física Nuclear da Universidade de Lisboa, P-1649-003 Lisboa, Portugal
}

(Received June 27, 2013)

\begin{abstract}
Background: The core excitation, being an important reaction mechanism, so far is not properly included in most calculations of three-body nuclear reactions.

Purpose: We aim to include the excitation of the core nucleus using an exact Faddeev-type framework for nuclear reactions in the three-body (core + neutron + proton) system.

Methods: We employ Alt, Grassberger, and Sandhas (AGS) integral equations for the three-particle transition operators and solve them in the momentum-space framework. The Coulomb interaction is included via the method of screening and renormalization.

Results: We calculate elastic, inelastic, and transfer reactions involving ${ }^{10} \mathrm{Be}$ and ${ }^{24} \mathrm{Mg}$ nuclear cores.

Conclusions: Important effects of the core excitation are found, often improving the description of the experimental data. In the neutron transfer reactions the core excitation effect is by far not just a simple reduction of the cross section by the respective spectroscopic factor. This indicates that widely used extraction of the spectroscopic factors from the ratio of the experimental and theoretical transfer cross sections is unreliable approach.

PACS numbers: 24.10.-i, 21.45.-v, 25.45.Hi, 25.40.Hs
\end{abstract}

Direct nuclear reactions of three-body nature provide an important test for models of nuclear dynamics. Widely studied examples are deuteron $(d)$ scattering from a stable nucleus $(A)$ and proton $(p)$ scattering from a weakly bound nucleus $(A n)$ consisting of a core $A$ and a neutron $(n)$. Elastic, inelastic, breakup, transfer, and charge-exchange reactions can be realized in such nuclear systems. A number of theoretical approaches has been developed and used for the analysis of these reactions, ranging from relatively simple distorted-wave Born approximation (DWBA), adiabatic wave approximation (ADWA) [1], and semiclassical eikonal approximation 2] to more sophisticated continuum discretized coupled channels (CDCC) method [3] and exact Faddeevtype scattering theory [4, 5]. The technical complexity of the latter precluded it from being used widely for the analysis of nuclear reaction data, but it was namely the Faddeev-type method that allowed to test the accuracy of the traditional nuclear reaction approaches and revealed their limitations in particular cases [6-8].

However, the standard technical implementations of the above-mentioned methods do not take explicitly into account the excitations of the core nucleus $A$ that may be an important reaction mechanism. Some of them, like DWBA and ADWA, assume that the core excitation effects simply lead to the reduction of transfer cross sections by a spectroscopic factor (SF) but the reliability of this approximation is unknown. Nevertheless, in last years several attempts have been made to include the core excitation into the three-body reaction dynamics. The extensions of the DWBA [9, 10] and CDCC methods 11, 12], the latter called XCDCC, were developed to calculate the breakup of one-neutron halo nucleus, however, with contradicting results. Another extension of CDCC with additional approximations was attempted in elastic and inelastic deuteron scattering [13]. Faddeevtype theory with separable potentials was proposed in
Ref. 14], however, without any numerical calculations.

On the other hand, a similar kind of scattering calculations with dynamic excitation of involved particles was developed in 90's for the three-nucleon $(3 N)$ system 15 17]. It includes the excitation of a nucleon into a $\Delta$ isobar via coupled-channel potential [18] in the framework of momentum-space symmetrized Alt, Grassberger, and Sandhas (AGS) equations [5] that are equivalent to Faddeev equations [4] but are formulated for the transition operators. While early calculations [15-17] relied on a separable representation of the interaction and were restricted to neutron-deuteron scattering, later developments include fully realistic non-separable coupledchannel potential with $\Delta$ isobar excitation [19, 20] and Coulomb interaction 21] using the method of screening and renormalization [22, 23]. The latter achievement allowed the application of the AGS equations also to threebody nuclear reactions in the $A+n+p$ system [6, 24, 25], albeit considering $A$ as an inert particle so far. It is the aim of the present work to overcome this limitation by developing exact Faddeev-type calculations for reactions in the $A+n+p$ system where the core nucleus $A$ with mass $m_{A}$ can be excited into $A^{*}$ with mass $m_{A^{*}}$ when interacting with nucleons; for simplicity we consider here only one excited state $A^{*}$ but the generalization to several excitations is straightforward. For this purpose we combine the strategy of Refs. [19, 20] and [6, 24, 25]. The developed method will be illustrated by the results for elastic, inelastic, and transfer reactions involving ${ }^{10} \mathrm{Be}$ and ${ }^{24} \mathrm{Mg}$ nuclear cores.

The three-particle scattering problem is formulated in the Hilbert space $\mathcal{H}_{g} \oplus \mathcal{H}_{x}$ with two sectors, where $\mathcal{H}_{g}$ contains states for $A+n+p$ free relative motion $\left|\mathbf{p}_{\alpha} \mathbf{q}_{\alpha}\right\rangle_{g}$ and $\mathcal{H}_{x}$ contains free $A^{*}+n+p$ states $\left|\mathbf{p}_{\alpha} \mathbf{q}_{\alpha}\right\rangle_{x}$. Here $\mathbf{p}_{\alpha}$ and $\mathbf{q}_{\alpha}$ are the three-particle Jacobi momenta in any of three possible configurations with spectator particle $\alpha$; the respective reduced masses will be denoted by $\mu_{\alpha}$ 
and $M_{\alpha}$. For brevity we suppress the dependence on spin quantum numbers. The extended free Hamiltonian $H_{0}$ in addition to kinetic energy operator includes also the intrinsic Hamiltonian of the core [10, 14] whose contribution is the mass difference relative to the $A+n+p$ threshold, i.e., $H_{0}\left|\mathbf{p}_{\alpha} \mathbf{q}_{\alpha}\right\rangle_{g}=\left(p_{\alpha}^{2} / 2 \mu_{\alpha}+q_{\alpha}^{2} / 2 M_{\alpha}\right)\left|\mathbf{p}_{\alpha} \mathbf{q}_{\alpha}\right\rangle_{g}$ and $H_{0}\left|\mathbf{p}_{\alpha} \mathbf{q}_{\alpha}\right\rangle_{x}=\left(p_{\alpha}^{2} / 2 \mu_{\alpha}+q_{\alpha}^{2} / 2 M_{\alpha}+\Delta m\right)\left|\mathbf{p}_{\alpha} \mathbf{q}_{\alpha}\right\rangle_{x}$ with $\Delta m=m_{A^{*}}-m_{A}$. The two sectors $\mathcal{H}_{g}$ and $\mathcal{H}_{x}$ are coupled by the core-nucleon interaction.

In such an extended Hilbert space the AGS scattering equations for three-particle transition operators $U_{\beta \alpha}$ have the standard form

$$
U_{\beta \alpha}=\bar{\delta}_{\beta \alpha} G_{0}^{-1}+\sum_{\gamma=1}^{3} \bar{\delta}_{\beta \gamma} T_{\gamma} G_{0} U_{\gamma \alpha} .
$$

Here $\bar{\delta}_{\beta \alpha}=1-\delta_{\beta \alpha}$ and $G_{0}=\left(E+i 0-H_{0}\right)^{-1}$ is the free resolvent at the available three-particle energy $E$ in the center of mass (c.m.) system; obviously, $G_{0}$ is diagonal in the two Hilbert sectors. The two-particle transition operator

$$
T_{\gamma}=v_{\gamma}+v_{\gamma} G_{0} T_{\gamma}
$$

for the pair $\gamma$ in the odd-man-out notation is derived from the respective two-particle potential $v_{\gamma}$; unless $\gamma$ corresponds to $n p$ pair, $v_{\gamma}$ and $T_{\gamma}$ couple the sectors $\mathcal{H}_{g}$ and $\mathcal{H}_{x}$. Obviously, the three-particle transition operators $U_{\beta \alpha}$ couple the two sectors too; their on-shell matrix elements $\left\langle\phi_{\beta}\left|U_{\beta \alpha}\right| \phi_{\alpha}\right\rangle$ between the respective asymptotic channel states are scattering amplitudes from which observables for all reactions allowed by the chosen Hamiltonian can be calculated. The two-cluster channel states $\left|\phi_{\alpha}\right\rangle$ with $\alpha=1,2,3$ are given by the respective twoparticle bound state wave function times the plane wave for the relative spectator-pair motion; the former has components in both sectors $\mathcal{H}_{g}$ and $\mathcal{H}_{x}$ if $\alpha$ corresponds to the core-nucleon pair.

The original AGS equations are formulated for shortrange potentials $v_{\gamma}$. However, as already mentioned, the long-range repulsive Coulomb force acting within one pair of particles can be included using the method of screening and renormalization [22, 23] in the AGS framework. The long-range part of the scattering amplitude is known analytically, whereas the Coulomb-distorted short-range part is obtained by solving the AGS equations with nuclear plus screened Coulomb potentials numerically and ensuring the convergence of the results with the screening radius. An efficient way to achieve this goal is proposed in Ref. [21]. The solution technique relies on a partial-wave decomposition and discretization of integrals using Gaussian quadratures with special (standard) weights for singular (non-singular) integrands. Thus, while the numerical techniques for solving the AGS equations with core excitation are taken over from Refs. [6, 19 21, 24], there are two novel aspects vis-a-vis those previous calculations relevant for practical implementation: i) The core excitation energy $\Delta m$, being $3.368 \mathrm{MeV}$ for ${ }^{10} \mathrm{Be}$ and $1.369 \mathrm{MeV}$ for ${ }^{24} \mathrm{Mg}$, is much smaller as compared to the $\Delta$-nucleon mass difference of nearly $300 \mathrm{MeV}$. Thus, while in Refs. [19, 20] the $\Delta$-isobar excitations are virtual with no $\Delta$ components in asymptotic channel states, the channels with the core in both ground $A$ and excited $A^{*}$ states are open in the considered energy regime. This implies also the presence of singularities in the momentum-space integral equation kernels in both sectors $\mathcal{H}_{g}$ and $\mathcal{H}_{x}$; they are treated by the real axis integration as described in detail in Refs. [17, 26]. ii) Typically, the core in the excited state has higher spin. In the examples of the present work, ${ }^{10} \mathrm{Be}$ and ${ }^{24} \mathrm{Mg}$, the spin/parity is $0^{+}$and $2^{+}$for $A$ and $A^{*}$, respectively. In this case the number of partial waves to be included in the sector $\mathcal{H}_{x}$ is larger by a factor of 5 as compared to $\mathcal{H}_{g}$, leading to increase of computer time by a factor of 25 . Thus, when including the core excitation the parallelization of calculations becomes mandatory.

Finally, we note that the present formulation of threebody reactions with core excitation is equivalent to the one of Ref. 14] except for a different way to include Coulomb force and no need for separable potentials.

The dynamic input to AGS equations are the potentials $v_{\gamma}$ for all three pairs. As the $n p$ interaction we take the realistic CD Bonn potential [27], in contrast to a simple Gaussian $n p$ potential used in DWBA and XCDCC calculations [10, 12]. The nucleon-core potentials are less constrained having many possible choices. In this work we do not aim to use the most sophisticated model but rather to isolate the effects of the core excitation. We therefore perform calculations with several models of $n A$ and $p A$ interactions:

a) The single particle (SP) model neglects the core excitation and uses the original Chapel Hill 89 (CH89) 28] optical potential (OP) for the $p A$ pair and for the $n A$ pair in the partial waves without bound states. The $n A$ potential in the partial waves with bound states is real with the strength adjusted to binding energy. As the OP parameters are energy dependent, various choices are possible for the energies at which $v_{N A}$ are to be calculated. For the ${ }^{10} \mathrm{Be}+n+p$ system where we are mostly interested in neutron transfer reactions we choose to fix $v_{p A}$ at the proton energy in the $p+{ }^{11} \mathrm{Be}$ channel and $v_{n A}$ at half the deuteron energy in the $d+{ }^{10} \mathrm{Be}$ channel. This is exactly the choice labeled FADD in Ref. 77]; the $n-{ }^{10} \mathrm{Be}$ binding potential is taken over from Ref. [7] as well. For $d+{ }^{24} \mathrm{Mg}$ elastic and inelastic scattering the CH89 OP taken at half the initial deuteron energy is used for both $p A$ and $n A$ pairs in all partial waves, i.e., the ${ }^{25} \mathrm{Mg}$ bound states are not supported. This choice corresponds to the one labeled FAGS in Ref. [8]. The respective strategies in fixing the OP parameters will be used also with other nucleon-core interaction models described below.

b) As in Refs. [9, 10, 12], the $n A$ and $p A$ interactions with the core excitation (CX) are obtained from the rotational model for the core 29]. It assumes that the core has a permanent quadrupole deformation characterized by the deformation parameter $\beta_{2}$. The radial 


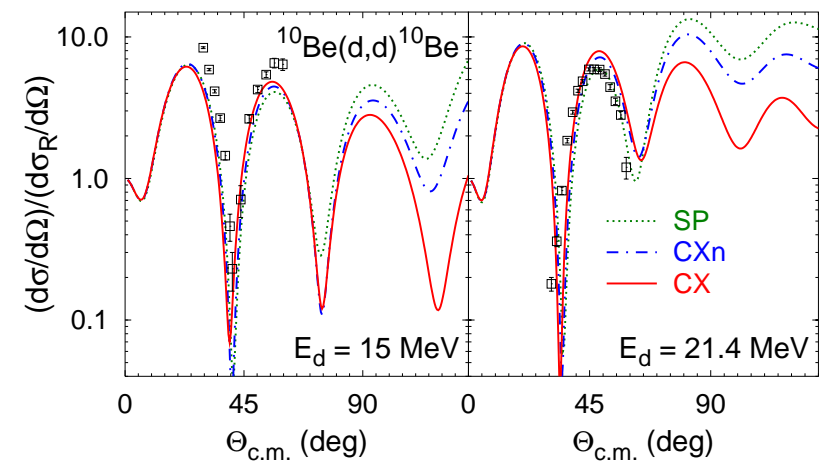

FIG. 1. (Color online) Differential cross section for $d+{ }^{10} \mathrm{Be}$ elastic scattering at $E_{d}=15$ and $21.4 \mathrm{MeV}$. Results including core excitation in both $n^{-}{ }^{10} \mathrm{Be}$ and $p_{-}{ }^{10} \mathrm{Be}$ interactions (solid curves), only in $n-{ }^{10} \mathrm{Be}$ interaction (dashed-dotted curves), and neglecting the core excitation (dotted curves) are compared with experimental data from Ref. [31].

dependence of the nucleon-core potential is given by the Woods-Saxon function $f(r, R, a)=\{1+\exp [(r-R) / a]\}^{-1}$ and, eventually, by its derivative, with the radius $R=$ $R_{0}\left(1+\beta_{2} Y_{20}(\hat{\xi})\right)$ depending on the quadrupole deformation and the internal core degrees of freedom $\hat{\xi}$ in the body-fixed frame. Obviously, this approach is equivalent to the replacement of the undeformed central potential $v_{N A}(r)$ by $v_{N A}\left(r-\delta_{2} Y_{20}(\hat{\xi})\right)$ where $\delta_{2}=\beta_{2} R_{0}$ is the deformation length. The resulting noncentral potential is expanded into multipoles as in Refs. [9, 10] with the $\lambda=2$ multipole term being responsible for the core excitation/deexcitation. In addition we include also the standard undeformed spin-orbit potential as provided by the CH89 parametrization [28]. The geometry parameters for the $n^{-10} \mathrm{Be}$ binding potential in the $1 / 2^{+}$ state are taken over from Ref. [29], i.e., $R_{0}=2.483 \mathrm{fm}$, $a=0.65 \mathrm{fm}$, and $\beta_{2}=0.67$, resulting $\delta_{2}=1.664 \mathrm{fm}$. The strengths of the central $V_{0}=-54.45 \mathrm{MeV}$ and spin-orbit $V_{\text {so }}=-8.5 \mathrm{MeV} \mathrm{fm}^{2}$ parts are slightly readjusted to reproduce better the ${ }^{11} \mathrm{Be}$ ground state energy of -0.504 $\mathrm{MeV}$. Its wave-function component with $s$-wave neutron coupled to the ground state $0^{+}$of ${ }^{10} \mathrm{Be}$ has the weight (SF) of 0.855 . The OP's for the $n A$ pair in other partial waves and for $p A$ pair are obtained by deforming the central part of the CH89 potential with $\delta_{2}=1.664 \mathrm{fm}$ for ${ }^{10} \mathrm{Be}$. For ${ }^{24} \mathrm{Mg}$ the values of $\beta_{2}$ range from 0.35 to 0.6 [30]; we therefore perform two CX calculations with $\beta_{2}=0.4$ and 0.6 , corresponding to $\delta_{2}=1.352$ and 2.028 $\mathrm{fm}$. In all cases the potentials are supplemented by the undeformed spin-orbit part of $\mathrm{CH} 89$.

c) To separate the effects of the core excitation due to interaction with the neutron and proton, for reactions involving ${ }^{10} \mathrm{Be}$ we perform also the calculations with hybrid model CXn, that uses the $p A$ potential from the SP model but the $n A$ potential from the CX model.

Results obtained with these interaction models for the $d+{ }^{10} \mathrm{Be}$ elastic scattering at $E_{d}=15$ and $21.4 \mathrm{MeV}$

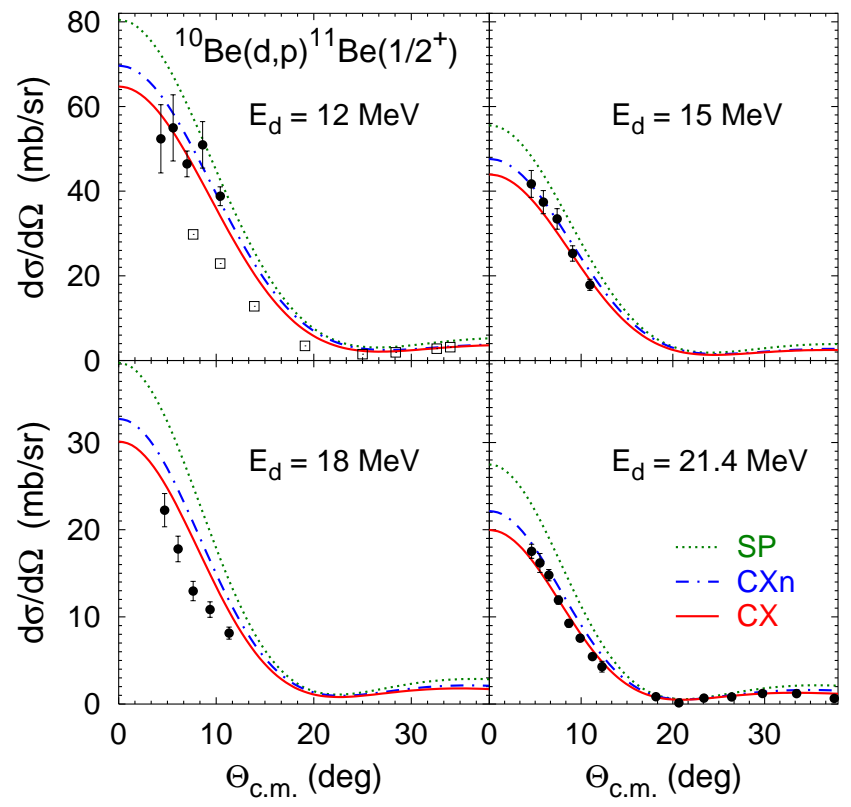

FIG. 2. (Color online) Differential cross section for the transfer reaction ${ }^{10} \mathrm{Be}(d, p){ }^{11} \mathrm{Be}$ at $E_{d}=12,15,18$, and $21.4 \mathrm{MeV}$ leading to the ground state $1 / 2^{+}$of ${ }^{11} \mathrm{Be}$. Curves as in Fig. 1 and experimental data from Refs. 31, 32].

deuteron lab energy are presented in Fig. 1. The differential cross section $d \sigma / d \Omega$ divided by the Rutherford cross section $d \sigma_{R} / d \Omega$ is shown as a function of the c.m. scattering angle $\Theta_{\text {c.m. }}$. The effect of the core excitation is most pronounced at large scattering angles $\Theta_{\text {c.m. }}>80^{\circ}$ where it reduces the cross section by a factor of 2 to 5 . In contrast, around $\Theta_{\text {c.m. }}=50^{\circ}$ where the experimental data from Ref. 31] are available, the core excitation slightly increases the cross section improving the agreement with data at $E_{d}=15$ but worsening at $21.4 \mathrm{MeV}$. The predictions of the CXn model usually lie between those of SP and CX, indicating that the core excitation effects due to $n-{ }^{10} \mathrm{Be}$ and $p-{ }^{10} \mathrm{Be}$ interactions are quite similar for the elastic scattering of deuteron.

The differential cross section for the deuteron stripping reaction ${ }^{10} \mathrm{Be}(d, p){ }^{11} \mathrm{Be}$ leading to the ground state $1 / 2^{+}$ of ${ }^{11} \mathrm{Be}$ is shown in Fig. 2 for $E_{d}=12,15,18$, and 21.4 $\mathrm{MeV}$. The most important core excitation effect is observed at small scattering angles where it decreases the cross section. Here the contribution of the core excitation in the $n^{-10} \mathrm{Be}$ pair is more sizable than in the $p-{ }^{10} \mathrm{Be}$ pair. With increasing energy the differential cross section decreases but the relative effect of the core excitation increases, leading to quite good agreement between CXn and CX predictions and experimental data from Ref. [31], except at $E_{d}=18 \mathrm{MeV}$. Note, however, that theoretical results vary smoothly with energy while experimental data exhibit an abrupt decrease from $E_{d}=15$ to $18 \mathrm{MeV}$. The data at $E_{d}=12 \mathrm{MeV}$ from Refs. 31] and [32] also seem to be inconsistent.

Consistently with the observed energy dependence, 


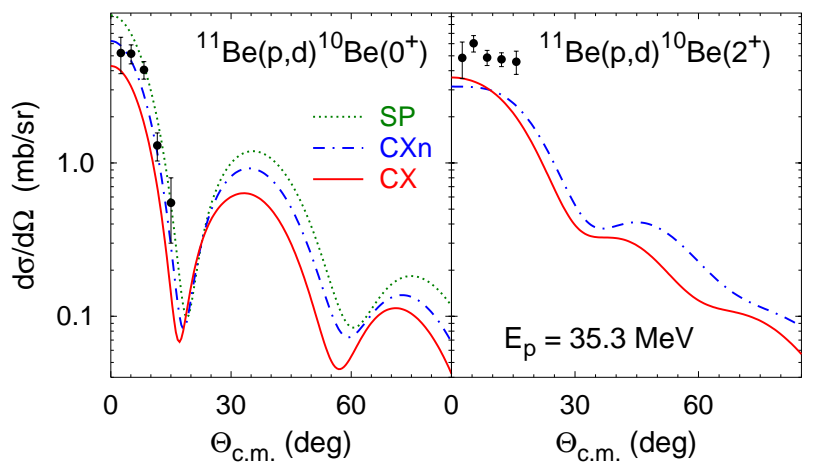

FIG. 3. (Color online) Differential cross section for the transfer reaction ${ }^{11} \mathrm{Be}(p, d){ }^{10} \mathrm{Be}$ at $E_{p}=35.3 \mathrm{MeV}$ leading to the ground $\left(0^{+}\right)$and excited $\left(2^{+}\right)$states of ${ }^{10} \mathrm{Be}$. Curves as in Fig. 1 and experimental data from Ref. [33].

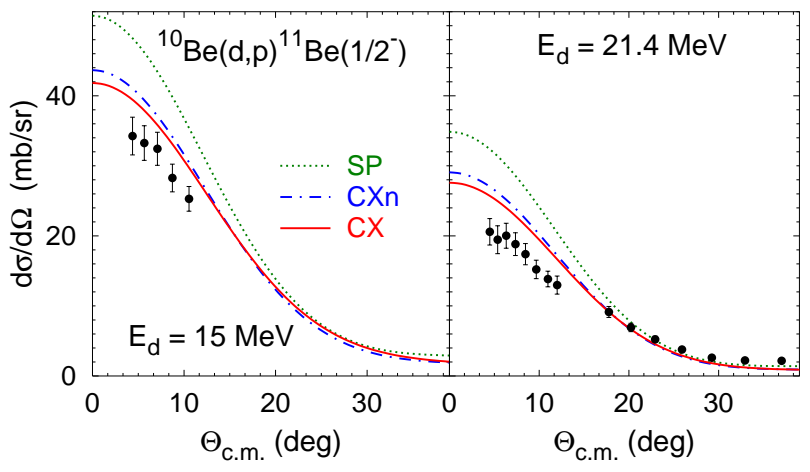

FIG. 4. (Color online) Differential cross section for the transfer reaction ${ }^{10} \mathrm{Be}(d, p){ }^{11} \mathrm{Be}$ at $E_{d}=15$ and $21.4 \mathrm{MeV}$ leading to the excited state $\left(1 / 2^{-}\right)$of ${ }^{11} \mathrm{Be}$. Curves as in Fig. 1 and experimental data from Refs. 32].

even larger core excitation effect is seen in Fig. 3 for the differential cross section of the deuteron pickup reaction ${ }^{11} \mathrm{Be}(p, d){ }^{10} \mathrm{Be}$ at proton lab energy $E_{p}=35.3 \mathrm{MeV}$, corresponding to $E_{d}=40.9 \mathrm{MeV}$ in the time-reverse reaction ${ }^{10} \mathrm{Be}(d, p){ }^{11} \mathrm{Be}$. Furthermore, in Fig. 3 we present also the the differential cross section for the reaction ${ }^{11} \mathrm{Be}(p, d){ }^{10} \mathrm{Be}$ leading to the ${ }^{10} \mathrm{Be}$ excited state $2^{+}$that is inaccessible in the calculations neglecting the core excitation. For both reactions in Fig. 3 the experimental data from Ref. [33] are underpredicted by CX and CXn calculations. Simultaneously we calculated also $p+{ }^{11} \mathrm{Be}$ elastic scattering (not shown) and found the core excitation effect to be insignificant.

The differential cross section for the neutron transfer reaction $\left.{ }^{10} \mathrm{Be}(d, p)\right)^{11} \mathrm{Be}$ leading to ${ }^{11} \mathrm{Be}$ in its excited

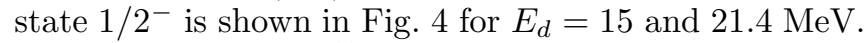
In this case a real $n-{ }^{10} \mathrm{Be}$ potential was used also in the $1 / 2^{-}$state with the strength $V_{0}=-49.62 \mathrm{MeV}$ resulting an excited bound state with the energy of -0.184 $\mathrm{MeV}$ and SF of 0.786 for the component with $p$-wave neutron coupled to the ground state of ${ }^{10} \mathrm{Be}$. Much like

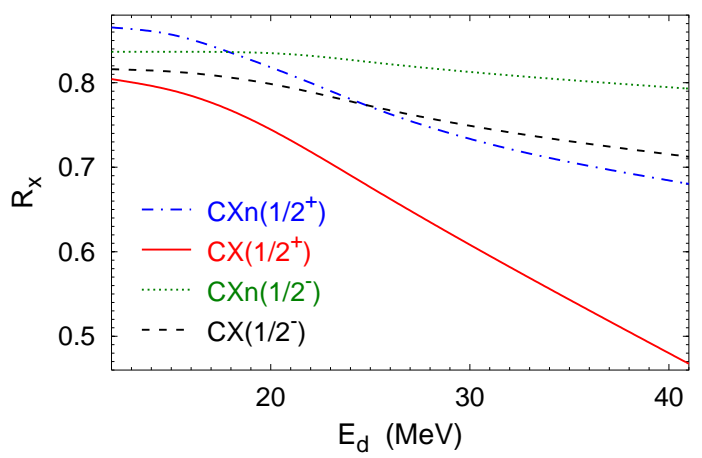

FIG. 5. (Color online) Ratios $R_{x}$ of differential cross sections for the transfer reactions ${ }^{10} \mathrm{Be}(d, p){ }^{11} \mathrm{Be}$ calculated with and without core excitation for $1 / 2^{+}$and $1 / 2^{-}$states of ${ }^{11} \mathrm{Be}$.

in Fig. 2, the core excitation effect is mostly pronounced at small scattering angles and decreases the cross section. It is even more strongly dominated by the $n-{ }^{10} \mathrm{Be}$ pair. However, unlike in Fig. 2. the experimental data from Refs. [31, 32] are overpredicted by the calculations.

In Fig. [5 we show the core excitation effect in the neutron transfer reactions on a finer scale. We observe that the ratios $R_{x}=(d \sigma / d \Omega)_{x} /(d \sigma / d \Omega)_{\mathrm{SP}}, x$ being either CX or CXn, depend only weakly on $\Theta_{\text {c.m. }}$ in the angular regime below the first minimum. We therefore take these ratios at $\Theta_{\text {c.m. }}=0^{\circ}$ and study their energy depen-

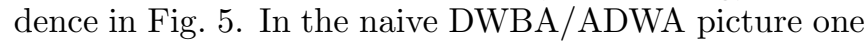
could expect these ratios to be equal to the respective SF for the $n-{ }^{10} \mathrm{Be}$ bound state wave-function component with the core in its ground state $0^{+}$, i.e., $0.855(0.786)$ for transfer to ${ }^{11} \mathrm{Be}$ ground (excited) state. Obviously, in a more sophisticated theory there are deviations from this naive picture as Fig. 5 demonstrates. For the ${ }^{11} \mathrm{Be}$ excited state $1 / 2^{-}$these deviations are moderate, up to $10 \%$. However, for the transfer to the $1 / 2^{+}$ground state of ${ }^{11}$ Be the ratios $R_{\mathrm{CX}}$ and $R_{\mathrm{CXn}}$ decrease with increasing energy and, especially $R_{\mathrm{CX}}$, significantly deviate from the SF value of 0.855 , reaching 0.47 at $E_{d}=41 \mathrm{MeV}$. Thus, these results indicate that extracting the SF from the ratio of the experimental and theoretical transfer cross sections as usually done in DWBA/ADWA is, in general, an unreliable approach, although in particular cases like ${ }^{10} \mathrm{Be}(d, p)^{11} \mathrm{Be}\left(1 / 2^{-}\right)$at low energies it may provide quite reasonable results. We emphasize that the present Faddeev-type reaction theory does not allow for a direct extraction of SF but reveals to what extent the employed dynamic model with core excitation predicting particular values for $\mathrm{SF}$ and reaction observables is compatible with given experimental data.

Finally we present results for elastic and inelastic scattering of deuterons on ${ }^{24} \mathrm{Mg}$; the latter reaction is only accessible in the dynamic model including core excitation. In Fig. 6] we show the respective differential cross sections at $E_{d}=70 \mathrm{MeV}$. In elastic scattering the core excitation effect is most visible at large angles where it decreases 


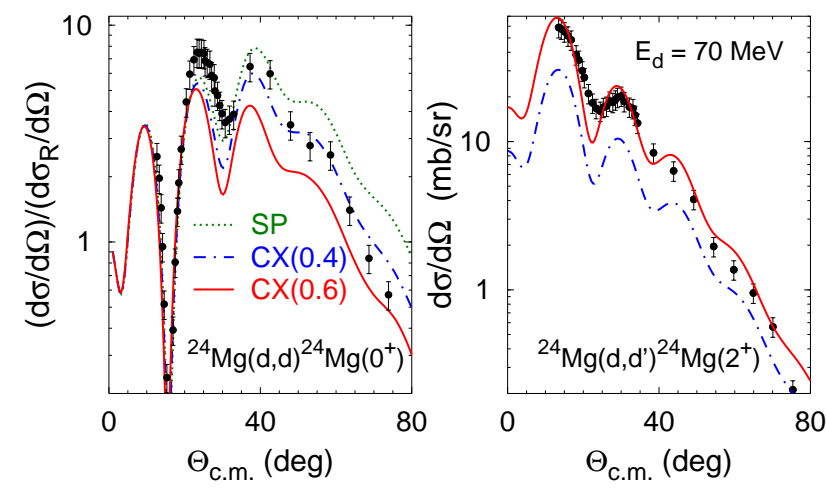

FIG. 6. (Color online) Differential cross section for $d+{ }^{24} \mathrm{Mg}$ elastic (left) and inelastic (right) scattering at $E_{d}=70 \mathrm{MeV}$. Results including core excitation with $\beta_{2}=0.4$ (dasheddotted curves) and $\beta_{2}=0.6$ (solid curves) and neglecting the core excitation (dotted curves) are compared with experimental data from Ref. [30].

the cross section, improving the description of the experimental data from Ref. [30] in the case of $\beta_{2}=0.4$. However, around the maximum at $\Theta_{\text {c.m. }}=50^{\circ}$ all calculations are below the data. The inelastic cross section data is underpredicted by theory nearly by a factor of 2 if one uses $\beta_{2}=0.4$ but is quite well described using $\beta_{2}=0.6$. The shape of the angular dependence with several maxima and minima is reproduced in both cases.

In summary, we performed calculations of elastic, inelastic, and transfer reactions in the nuclear three-body system $A+p+n$ including the excitation of the core nucleus $A$. Exact scattering equations in the AGS form were solved with the Coulomb interaction included via the method of screening and renormalization. Example results for ${ }^{10} \mathrm{Be}$ and ${ }^{24} \mathrm{Mg}$ cores were presented. Important effects of the core excitation were found, in most cases improving the description of the experimental data. Furthermore, we demonstrated that in the neutron transfer reactions the core excitation effect cannot be simply simulated by the reduction of the cross section according to the respective $\mathrm{SF}$ as assumed in DWBA/ADWA calculations. This deviation from DWBA/ADWA is not surprising given the fact that the employed Faddeev-type reaction theory is an exact one and includes interactions between the three involved particles up to all orders. This finding also indicates that extracting the SF from the ratio of experimental and theoretical transfer cross sections is unreliable approach. The present Faddeev-type reaction theory does not allow for a direct extraction of $\mathrm{SF}$ but enables a rigorous test of the employed dynamic model. In this respect further improvements are possible, e.g., readjusting the parameters of the deformed optical potentials to get a better description of the twobody data, and using nonlocal potentials with the core excitation since the nonlocality is known to be important in transfer reactions [25, 34]. We hope that the present work, demonstrating the feasibility of exact calculations with core excitation and its importance, will stimulate the development of more sophisticated and precise interaction models.

The author thanks A. M. Moro for discussions and comparison of two-body results.
[1] R. C. Johnson and P. J. R. Soper, Phys. Rev. C 1, 976 (1970).

[2] D. Baye et al., Phys. Rev. C 79, 024607 (2009).

[3] N. Austern et al., Phys. Rep. 154, 125 (1987).

[4] L. D. Faddeev, Zh. Eksp. Teor. Fiz. 39, 1459 (1960).

[5] E. O. Alt, P. Grassberger, and W. Sandhas, Nucl. Phys. B2, 167 (1967).

[6] A. Deltuva et al., Phys. Rev. C 76, 064602 (2007).

[7] F. M. Nunes and A. Deltuva, Phys. Rev. C 84, 034607 (2011).

[8] N. J. Upadhyay, A. Deltuva, and F. M. Nunes, Phys. Rev. C 85, 054621 (2012).

[9] R. Crespo, A. Deltuva, and A. M. Moro, Phys. Rev. C 83, 044622 (2011).

[10] A. M. Moro and R. Crespo, Phys. Rev. C 85, 054613 (2012).

[11] N. C. Summers, F. M. Nunes, and I. J. Thompson, Phys. Rev. C 74, 014606 (2006).

[12] N. C. Summers and F. M. Nunes, Phys. Rev. C 76, 014611 (2007).

[13] P. Chau Huu-Tai, in Proceedings of INPC07 (Elsevier, Nucl. Phys. A, 2008), Vol. II, p. 483.

[14] A. M. Mukhamedzhanov, V. Eremenko, and A. I. Sattarov, Phys. Rev. C 86, 034001 (2012).
[15] S. Nemoto et al., Few-Body Systems 24, 213 (1998).

[16] S. Nemoto et al., Few-Body Systems 24, 241 (1998).

[17] K. Chmielewski et al., Phys. Rev. C 67, 014002 (2003).

[18] C. Hajduk, P. U. Sauer, and W. Strueve, Nucl. Phys. A405, 581 (1983).

[19] A. Deltuva, K. Chmielewski, and P. U. Sauer, Phys. Rev. C 67, 034001 (2003).

[20] A. Deltuva, R. Machleidt, and P. U. Sauer, Phys. Rev. C 68, 024005 (2003).

[21] A. Deltuva, A. C. Fonseca, and P. U. Sauer, Phys. Rev. C 71, 054005 (2005); 72, 054004 (2005).

[22] J. R. Taylor, Nuovo Cimento B 23, 313 (1974); M. D. Semon and J. R. Taylor, Nuovo Cimento A 26, 48 (1975).

[23] E. O. Alt and W. Sandhas, Phys. Rev. C 21, 1733 (1980).

[24] A. Deltuva and A. C. Fonseca, Phys. Rev. C 79, 014606 (2009).

[25] A. Deltuva, Phys. Rev. C 79, 021602(R) (2009).

[26] A. Deltuva, Ph.D. thesis, University of Hannover, 2003, http://edok01.tib.uni-hannover.de/edoks/e01dh03/374454701.pdf

[27] R. Machleidt, Phys. Rev. C 63, 024001 (2001).

[28] R. L. Varner et al., Phys. Rep. 201, 57 (1991).

[29] F. Nunes et al., Nucl. Phys. A 609, 43 (1996).

[30] A. Kiss et al., Nucl. Phys. A 262, 1 (1976).

[31] K. T. Schmitt et al., Phys. Rev. Lett. 108, 192701 (2012). 
[32] D. L. Auton, Nucl. Phys. A157, 305 (1970).

[33] J. Winfield et al., Nucl. Phys. A683, 48 (2001).

[34] A. Deltuva, Phys. Rev. C 79, 054603 (2009). 